(Phys. Rev. 168, 209; 1968) that one way of exciting vortex waves might be to subject the line to a transverse oscillatory electric field while ions were travelling along it. This resonant excitation of vortex waves would have a corresponding effect on the motion of the ion. To achieve it, several conditions must be fulfilled. In particular, the ions should be moving along the line at the same speed as the propagation velocity of the vortex wave corresponding to the frequency of the applied electric field. Halley and Osermeier were subsequently able to calculate in detail ( $J$. Low Temperature Phys. 26, 877; 1977) how the velocity of the ion might be expected to vary with the d.c. electric field propelling them along the line. They predicted that there should be a kink in the velocity-field characteristic at a certain critical velocity, which depends on the frequency of the transverse oscillatory electric field.

Ashton and Glaberson have now performed this experiment, using a rotating ${ }^{3} \mathrm{He}$ cryostat in which the ${ }^{4} \mathrm{He}$ sample can be cooled to $0.3 \mathrm{~K}$ while being rotated at speeds of up to $10 \mathrm{rad} \mathrm{s}^{-1}$. The transverse electric field was circularly polarised, so that resonant vortex wave production was expected to occur for only one polarisation for a given sense of cryostat rotation. Their experimental results are shown in Fig. 2. It is quite clear that the radiofrequency (RF) electric field has a considerable influence on the ionic velocity $V$, and that entirely different effects are obtained when the applied field is changed from a clockwise (CW) to a counterclockwise (CCW) sense relative to

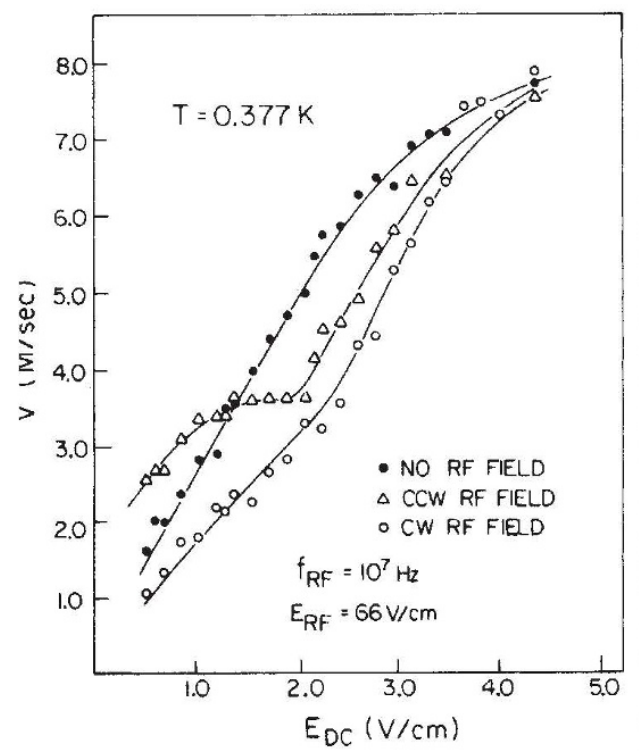

Fig. 2 Measurements of the ionic velocity $V$ along a vortex line as a function of the longitudinal electric field $E_{\mathrm{DC}}$ : for zero applied radiofrequency (RF) field; and with the latter field polarised clockwise $(\mathrm{CW})$ or counterclockwise (CCW) relative to the rotation of the experimental cell. The anomalous plateau and kink at about $3.6 \mathrm{~m}$ $\mathrm{s}^{-1}$ in the CCW curve is interpreted as evidence of a coupling of the ions to vortex waves.

the rotation of the cryostat. An anomalous kink and plateau occurs in the velocity at about $3.6 \mathrm{~m} \mathrm{~s}^{-1}$, in tolerable agreement with a theoretical prediction of $3 \mathrm{~m} \mathrm{~s}^{-1}$ for the RF frequency being used $(10 \mathrm{MHz})$. This result can be regarded as a vindication of the principal conclusions of the Halley and Ostermeier theory. But the theory does not give a satisfactory account of the detailed shape of the velocity-field curve; nor is it able to explain the reduced values of $V$ found in the $\mathrm{CW}$ rotation.

A good deal more work, both theoretical and experimental, will be required to sort out the details. Even on the basis of the present results, however, it seems clear that high frequency vortex waves really do occur in superfluid ${ }^{4} \mathrm{He}$.

\title{
The cladistic debate continued
}

The critical view of the cladistic method of classification taken by L.B. Halstead in his report of a meeting on Vertebrate Palaeontology published in News and Views some months ago (276, 759; 1978) and the rejoinder by a group of supporters of 'cladism' 277 , 175 ; 1979) provoked a large correspondence, a selection of which is published below.

There can be few disagreements on methodology in the recent history of biology which have been pursued with such passion by the protagonists, while leaving biologists not involved (in this case in systematics) so unmoved by, or even ignorant of, all the fuss. There is fruitful territory for psychologists here, but I would like to confine myself to the methodological rather than the theological aspects of the affair.

The fundamental tenet of cladism, is that in systematic biology the reconstruction of phylogeny is logically prior to the construction of a classification and that the latter is a redundant expression of the former. Unlike all other taxonomists, the cladists characterise phylogeny solely as the result of speciation or cladogeneses (the splitting of one species into two or more in evolution). They ignore the results of evolutionary change within evolving species (phyletic evolution or anagenesis). Furthermore in reconstructing phylogeny they allow division only by dichotomy: that is, speciation, according to them, is always the splitting of one species into two daughter species, sister species of one another. Primitive cladists held this as a dogma, which, with our current knowledge of evolutionary genetics, is patently absurd. Sophisticated advocates use it only as an operational principle. Nevertheless the rationale of the method depends on evolution by speciation which results in reproductive isolation between sexually reproducing organisms. Cladistic classification is therefore inapplicable to non-sexual organisms and will presumably never be used for microorganisms such as Protozoa, bacteria and viruses.

The recognition of sister species (or groups of higher rank) is by shared derived characters unique to the sister groups and thus presumed present in their (always hypothetical) unique common ancestral species. Shared primitive characters, those also present in collateral groups outside the pair, are ignored. Unfortunately if a large number of character states is considered, as it should be by any competent taxonomist, contradictions will arise due to convergently evolved characters mimicking true synapomorphies (shared derived ones). The method of resolving this difficulty destroys the whole rationale of the method of recognition of sister groups. The inconsistencies are resolved by using the principle of parsimony. By parsimony cladists mean that the sister-grouping which yields the smallest ('most parsimonious') number of contradictory characters compared with the number of apparent shared derived characters, of those the cladist has studied, is the correct one. This preposterous assumption could only be true if, first, an organism (be it lungfish, salmon or cow) could be analysed into a finite number of discrete characters, and, second, if a competent cladistic taxonomist could be reasonably sure that the tiny number of shared characters he can study in that organism has a similar ratio of true derived to convergent characters as does the class of all its characters.

Admittedly, traditional and also phenetic ('numerical') taxonomists use parsimony in an informal way, but they also use the criterion of predictability, perfectly described in the last century by John Stuart Mill, as an extrinsic criterion to test their classifications. Despite the fact that many cladists pay lip-service to the hypothetico-deductive method usually associated with the name of Sir Karl Popper, their only test of a cladogram appears to be a comparison, based on parsimony, with other contradictory cladograms. It is perhaps for this reason that a number of incompatible schemes for the mutual relationships of the major groups of fish-like vertebrates have been proposed by cladists, without any successful criterion within the system to choose between them.

At the risk of earning the opprobrium of your cladistic correspondents, I should like to end by pointing out that the problem I have touched on was well known to that very pre-Darwinian taxonomist Aristotle. In de Partibus Animalium (Book 1, paragraphs 2-4) he notes that a dichotomous classification based on a single character state will inevitably lead to an artificial classification (as it did recently when one eminent palacontologist used it in the fundamental division of the Class Mammalia), but that the use of several character states leads to contradictions which cannot be resolved.

A.L. PANCHEN

Department of Zoology,

The University, Newcastle-upon-Tyne, UK. 